Sādhanā Vol. 29, Part 5, October 2004, pp. 525-533. @ Printed in India

\title{
High speed forging of solid powder discs of large slenderness ratio
}

\author{
R K RANJAN $^{1}$ and S KUMAR ${ }^{2}$ \\ ${ }^{1}$ Applied Mechanics Department, and ${ }^{2}$ Production Engineering Department, Birla \\ Institute of Technology, Mesra, Ranchi 835 215, India \\ e-mail rkranjanbit@yahoo.com
}

MS received 30 July 2003; revised 13 January 2004

\begin{abstract}
The paper reports an investigation into the forging of a solid powder circular disc with large slenderness ratio $(L / D)$ between two flat dies at high speed. The deformation pattern during the operation is influenced by many factors, which interact with one another in a complex manner. The decisive factors are the interfacial conditions, initial relative density of the preform and the geometry of the preform. An attempt has been made to determine the die pressures developed during such forging, using an upper bound approach. The results so obtained are presented graphically and discussed critically to illustrate the interaction of various process parameters involved.
\end{abstract}

Keywords. Slenderness ratio; preform; interfacial friction law.

\section{Introduction}

During the last few years metal-powder components have assumed an important position in industry, as they are being used successfully in a wide range of applications. Both the mechanical and metallurgical properties of metal-powder components compare favourably with those of wrought materials (Ramakrishnan 1980). Bulk processing of metal-powder preforms is a convenient method of reducing or eliminating the porosity from conventional powder metallurgy products. The process is attractive because it avoids a large number of operations, high scrap losses and high-energy consumption associated with the conventional manufacturing processes such as casting, machining etc. In this new technology, sintered porous powder preforms are used as starting materials in metal forming processes. Metalpowder products manufactured by this new technology are comparable and in some cases even superior to cast and wrought products.

The deformation pattern during the forging of metal-powder preforms is different from conventional wrought metal forging and the following characteristics of porous materials undergoing deformation must be taken into account:

- A change in density occurs during plastic deformation;

A list of symbols is given at the end of the paper 


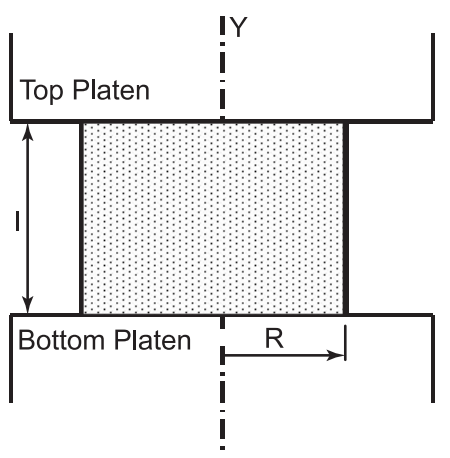

Figure 1. Forging assembly.

- The yielding of porous metals is not completely insensitive to the hydrostatic stress imposed during processing.

Although a considerable amount of work has been reported recently on the various technological aspects of the industrial processing of metal-powder performs (Sutradhar et al 1995, Jha \& Kumar 1983, 1996, 1997; Agrawal et al 1999; Kumar et al 2001), no systematic attempt has been made so far to study the processing load and deformation characteristics during high speed forging of circular discs of large slenderness ratio i.e. $l / D$ (where $l=$ height of the preform and $D=$ its diameter). Moreover, interest in high speed has grown considerably because of the introduction of new and fast deformation processes. Since speed is an important factor, attention should be paid towards the effects of inertial forces. In a fast deformation process, the flow of material is greatly influenced by interfacial conditions and factors related to the forging, such as deformation speed and contact time under load. Prediction of occurrence of defects requires understanding of the mechanics of deforming bodies and knowledge of strain and strain rate distributions that take place during deformation.

This paper reports investigation into the forging of circular preforms with large slenderness ratios, between two flat dies (figure 1). In this forming operation, the nature of the flow of material is not completely understood, and for actual cases an exact formulation of velocity model cannot be made. The main objective of this paper is to study the effect of slenderness ratio of the preform on the deformation pattern. It is expected that the present work will be of great importance for the assessment of die loads during forging of such preforms at high speeds.

\section{Interfacial friction law}

Friction condition between deforming tool and work piece in metal forming are of the greatest importance concerning a number of factors such as force and mode of deformation, properties of the finished specimen and resulting surface roughness. The relative velocity between the work piece material and the die surface together with high interfacial pressure and/or deformation modes will create the conditions essential for adhesion in addition to sliding (Deryagin 1952).

The pattern of the metal flow during the forging of a metal-powder preform is such that there exists two zones, an inner one where no relative movement between work piece and die occurs (the sticking zone) and an outer zone where sliding occurs. Therefore, the appropriate friction laws for particular condition is:

$$
\tau=\mu\left[p_{a v}+\rho_{0} \phi_{0}\left\{1-\left(R_{m}-R\right) /{ }^{n} R_{0}\right\}\right],
$$


where $r$ denotes the sticking zone radius and which may be approximated by the relation given by Rooks (1974) and $n \gg 1$.

\section{Yield criteria and plastic deformation of powder preforms}

In an investigation of the plastic deformation of sintered metal-powder preforms, it is clear that change in volume occurs due to porosity. A preform with a high relative density yields at a relative high stress whereas a low relative density preform yields at a relatively low stress. Even hydrostatic stress can cause sintered metal-powder preforms to yield, as the yield surface is closed on the hydrostatic stress axis. Density distribution also does not seem to be uniform throughout, being high in the central region and low at the edges. Density distribution is more uniform for a smaller coefficient of friction and for a higher initial-density preform.

Tabata \& Masaki (1977) proposed the following yield criterion for porous metal-powder preforms:

$$
\rho^{k}=\sqrt{3 J_{2}^{\prime}} \pm 3 \eta \sigma_{m}
$$

The negative sign is taken for $\sigma_{m} \leqslant 0$ and the positive sign is taken for $\sigma_{m}>0$.

Figure 2 shows that the yield surface for a porous metal-powder preform given by the above equation consists of two cones, the height of the cones increasing with increasing $\rho$. When $\rho=1$, i.e. for a pore-free metal, the cone becomes a cylinder and the equation reduces to the Von Mises yield criterion.

The values of $\eta$ and $k$ were determined experimentally from simple compression and tension tests on sintered copper-powder performs (Tabata and Masaki 1977) as

$$
\begin{aligned}
& \eta=0 \cdot 54(1-\rho)^{1 \cdot 2}, \text { for } \sigma_{m} \leqslant 0, \\
& \eta=0 \cdot 55(1-\rho)^{0.83}, \text { for } \sigma_{m}>0 \text { and } k=2 .
\end{aligned}
$$

For the axisymmetric condition the yield criterion reduces to (Tabata \& Masaki 1975)

$$
\sigma_{1}=\left[\rho^{k} \sigma_{0} /(1-2 \eta)\right]+[(1+\eta) /(1-2 \eta)] \sigma_{2} .
$$

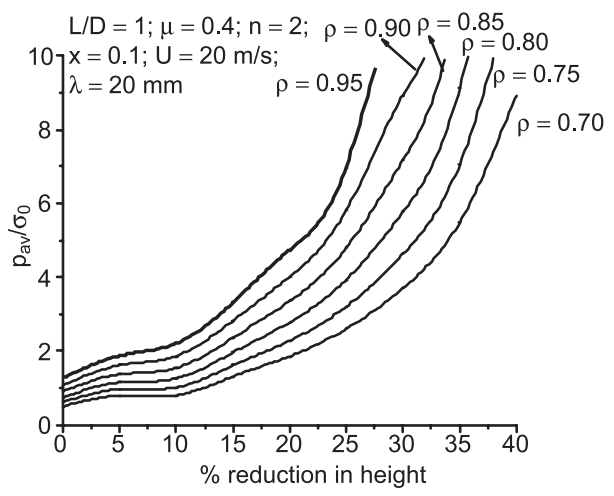

Figure 2. Variation of relative average forging pressure with percentage reduction in height at different initial relative densities. 


\section{Velocity field and strain rate}

The velocity field for the case under consideration is

$$
\begin{aligned}
U_{R} & =[(1-2 \eta) U R /(1+\eta) L][1+a \sin (2 \pi y / \lambda)], \\
U_{y} & =U\left[(1-(2 y / L))+\frac{a}{N \pi}(\cos (2 \pi y / \lambda)-\cos 2 N \pi)\right] ; \quad U_{\theta}=0,
\end{aligned}
$$

where

$$
a=-N \pi / 2 \text { for } N=L / \lambda=1 / 2,3 / 2, \ldots .
$$

The strain rates as calculated from (4) are

$$
\begin{aligned}
\varepsilon_{R} & =[(1-2 \eta) U /(1+\eta) L][1+a \sin (2 \pi y / \lambda)], \\
\varepsilon_{y} & =-(2 U / L)[1+a \sin (2 \pi y / \lambda)], \\
\varepsilon_{\theta} & =[(1-2 \eta) U /(1+\eta) L][1+a \sin (2 \pi y / \lambda)], \\
\varepsilon_{y R} & =[-(1-2 \eta) U R \pi a /(1+\eta) L \lambda] \cos (2 \pi y / \lambda), \\
\varepsilon_{\theta R} & =\varepsilon_{\theta y}=0 .
\end{aligned}
$$

The relationship of volume change between the strain rates is given as (Tabata \& Masaki 1975)

$$
\varepsilon_{R}+[(1-2 \eta) / 2(1+\eta)] \varepsilon_{y}=0 .
$$

\section{Internal power of deformation}

The internal power of deformation is given by-

$$
W_{i}=\frac{2}{\sqrt{3}} \sigma_{0} \int_{v}\left[\frac{1}{2}\left(\varepsilon_{R}^{2}+\varepsilon_{\theta}^{2}+\varepsilon_{y}^{2}\right)+2 \varepsilon_{R \theta}^{2}\right]^{1 / 2} \mathrm{~d} V
$$

where $\mathrm{d} V=2 \pi$ R.dR.dy.

Using the value of strain rates from (5), integration gives-

$$
W_{i}=\frac{4 \pi(1-2 \eta) U \sigma_{0}}{\sqrt{3}(1+\eta) L} \sqrt{1+\frac{2(1-2 \eta)^{2}}{(1+\eta)^{2}}} \cdot R_{0}^{2}\left[L+\frac{a \lambda}{2 \pi}-\frac{a \lambda}{2 \pi} \cos 2 N \pi\right] .
$$

\section{Energy dissipation due to friction}

The friction loss $W_{f}$ is given by-

$$
W_{f}=\int_{S} \tau|\Delta v| d s
$$


where $|\Delta v|$ is the velocity difference between platens and the cylinder in the direction of shear and parallel to the platen surface, i.e.

$$
|\Delta v|=[(1-2 \eta) /(1+\eta)] \cdot(U R / L), \text { and } d s=2 \cdot 2 \pi \mathrm{RdR} .
$$

From (1) and (8), after integration we get

$$
\begin{aligned}
W_{f} & =\frac{4 \pi(1-2 \eta) \mu U R_{0}^{3}}{3(1+\eta) L}\left[p_{a v}+\rho_{0} \phi_{0}\left\{1-\frac{R_{m}}{n R_{0}}+\frac{3}{4 n}\right\}\right], \\
\rho_{0} \phi_{0} & =x \cdot p_{a v} \text { and } x=0 \cdot 1,0 \cdot 2,0 \cdot 3, \ldots
\end{aligned}
$$

\section{Energy dissipation due to inertia force}

Energy dissipation is given by

$$
\begin{aligned}
W_{a}= & \frac{\rho_{r}}{g} \int_{v} a_{i} U_{i} d v=\frac{\rho_{r}}{g} \int_{0}^{R_{0}} \int_{0}^{L}\left(a_{R} U_{R}+a_{\theta} U_{\theta}+a_{y} U_{y}\right) 2 \pi R d R d y, \\
a_{R}= & U_{R} \frac{\partial U_{R}}{\partial R}+U_{y} \frac{\partial U_{R}}{\partial y}+\frac{\partial U_{R}}{\partial t}, \quad a_{y}=U_{y} \frac{\partial U_{y}}{\partial y}+\frac{\partial U_{y}}{\partial t}, \\
a_{R}= & \frac{(1-2 \eta)^{2} U^{2} R}{(1+\eta)^{2} L^{2}}\left[1+a \sin \frac{2 \pi y}{\lambda}\right]^{2}+\frac{(1-2 \eta) U^{2} R}{(1+\eta) L}\left[\left(1-\frac{2 y}{L}\right)\right. \\
+ & \left.\frac{a}{N \pi}\left(\cos \frac{2 \pi y}{\lambda}-\cos 2 N \pi\right)\right] \times \frac{2 a \pi}{\lambda} \cos \frac{2 \pi y}{\lambda}+\frac{(1-2 \eta) \dot{U} R}{(1+\eta) L} \\
& {\left[1+a \sin \frac{2 \pi y}{\lambda}\right], } \\
& {\left[\left(1-\frac{2 y}{L}\right)+\frac{a}{N \pi}\left(\cos \frac{2 \pi y}{\lambda}-\cos 2 N \pi\right)\right]\left[-\frac{2 U}{L}\left(1+a \sin \frac{2 \pi y}{\lambda}\right)\right] } \\
a_{y}= & +\dot{U}\left[\left(1-\frac{2 y}{L}\right)+\frac{a}{N \pi}\left(\cos \frac{2 \pi y}{\lambda}-\cos 2 N \pi\right)\right] .
\end{aligned}
$$

Neglecting $\dot{U}=\partial U / \partial t$ and by integrating and simplifying (10), we can get

$$
\begin{aligned}
W_{a}= & U^{3} \frac{\rho_{r}}{g} R^{2}\left[\frac{(1-2 \eta)^{3} R^{2}}{2(1+\eta)^{3} L}\left\{\frac{\lambda \pi}{L}+\frac{a^{2}}{4 N}\right\}-2+\frac{4 L}{\lambda(\lambda-1)}-\frac{8 L^{2}}{3 \lambda\left(\lambda-L^{2}\right)}\right. \\
& \left.+\frac{a^{2} L \pi}{\lambda\left(N-\pi^{2}\right)}-\frac{a^{2}}{N^{2}-\pi^{2}}\right] .
\end{aligned}
$$

\section{Die load}

For plastic deformation of a metal-powder the external power $\stackrel{*}{J}$ supplied by the platens is given as

$$
\stackrel{*}{J}=W_{i}+W_{f}+W_{a}+W_{t} .
$$


The first term on the right-hand side denotes the rate of internal energy dissipation $W_{i}$, the second term denotes the frictional shear energy losses $W_{f}$, the third term denotes the energy dissipation due to inertia forces $W_{a}$, and the last term covers power supplied by predetermined body tractions $W_{t}$. In this case no external surface traction is stipulated. Therefore, $W_{t}=0$.

Now the external power $J$ supplied by the press for entire hexagonal shape through the platen is -

$$
\begin{aligned}
& \stackrel{*}{J}= \int F_{i} U_{i} d s=P U, \text { and } P=p_{a v} \cdot 2 \pi R_{0}, \\
& \frac{p_{a v}}{\sigma_{0}}= {\left[1-\frac{2(1-2 \eta) \mu}{3(1+\eta) L}\left\{1+x\left(1-\frac{R_{m}}{n R_{0}}+\frac{3}{4 n}\right)\right\} R_{0}^{2}\right]^{-1} } \\
& {\left[\begin{array}{l}
\frac{2 \pi(1-2 \eta) U \sigma_{0}}{\sqrt{3}(1+\eta) L}\left(1+\frac{2(1-2 \eta)^{2}}{(1+\eta)^{2} L}\right)^{1 / 2} \cdot R_{0}\left(L+\frac{a \lambda}{2 \pi}-\frac{a \lambda}{2 \pi} \cos 2 N \pi\right) \\
+U^{2} \frac{\rho_{r}}{2 \pi g} R\left\{\frac{(1-2 \eta)^{3} R^{2}}{2(1+\eta)^{3} L}\left(\frac{\lambda \pi}{L}+\frac{a^{2}}{4 N}\right)-2+\frac{4 L}{\lambda(\lambda-1)}\right.
\end{array}\right.} \\
&\left.-\frac{8 L^{2}}{3 \lambda\left(\lambda-L^{2}\right)}+\frac{a^{2} L \pi}{\lambda\left(N-\pi^{2}\right)}-\frac{a^{2}}{N^{2}-\pi^{2}}\right\}
\end{aligned}
$$

\section{Results and discussions}

Figure 2 shows the variation of relative forging pressure with percentage reduction in height of the preform at particular values of coefficient of friction, $\lambda$ (wavelength) and velocity of the ram. In a powder metal-forming operation, there are two processes that occur simultaneously, i.e. compaction and deformation. Initially, compaction dominates; so relative average pressure curve increases slowly. After the compaction phase, deformation dominates and a steep slope is observed. Material at first flows mainly in the direction of punch movement, with little lateral flow. As the density increases, lateral flow increases. In the final stage of deformation, the lateral flow approach the spreading behaviour of pore-free material. Lateral/spreading

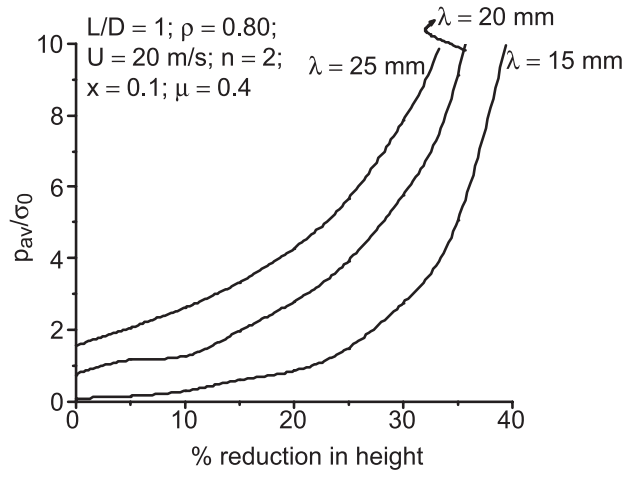

Figure 3. Variation of relative average forging pressure with percentage reduction in height of the preform for different value of $\lambda$. 


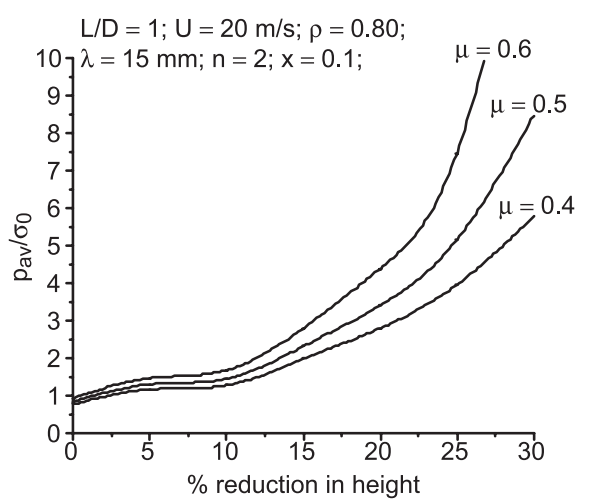

Figure 4. Variation of relative average forging pressure with percentage reduction in height of the preform for different value of coefficient.

increases with increasing initial density of preform. An increase in wavelength needs more relative average forging pressure (figure 3 ). From figure 4, it can be seen that relative average pressure increases with increase in coefficient of friction. Actually, during the compression of a metal-powder-preform, the compressive force gradually increases the relative density, which is directly proportional to the real area of contact. The real area of contact grows and approaches the apparent one as the force increases, which in turn increases the relative density. From this figure, it can be seen that for a particular value of coefficient of friction, the curves show an asymptotic nature (when $\rho \approx 1$ ) at a particular value of percentage reduction in the height of the preform. Hence, the curve indicates a limit of deformation for a particular set of inputs. From figure 5, it is observed that relative average forging pressure decreases with increase in the value of slenderness ratio $(L / D)$. Preforms having larger values of $L / D$ ratio show buckling action. They fail on large deformation of the preforms. This is why relative average pressure decreases as $L / D$ ratio increases. Figure 6 shows the variation of relative average pressure increases with increase in the value of ram velocity. However, ram velocity limits the possible reduction ratio of the preform, it can be seen from the nature of the graphs. Figure 7 shows the effect of slenderness ratio and ram velocity on relative average forging pressure. It is seen that as $L / D$ increases the forging pressure decreases and for a particular value of $L / D$, it increases with ram velocity.

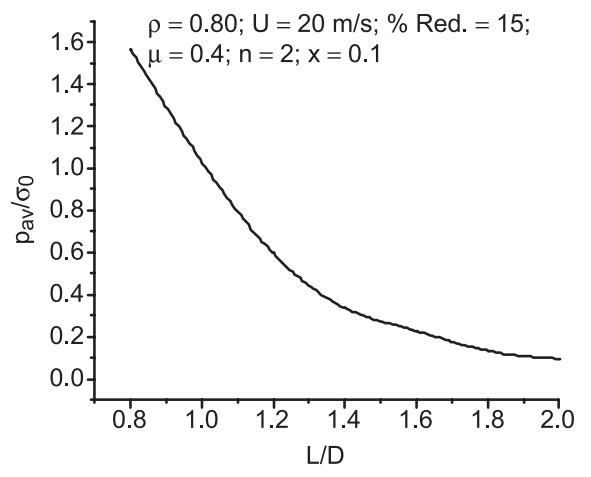

Figure 5. Variation of relative average pressure with respect to slenderness ratio. 


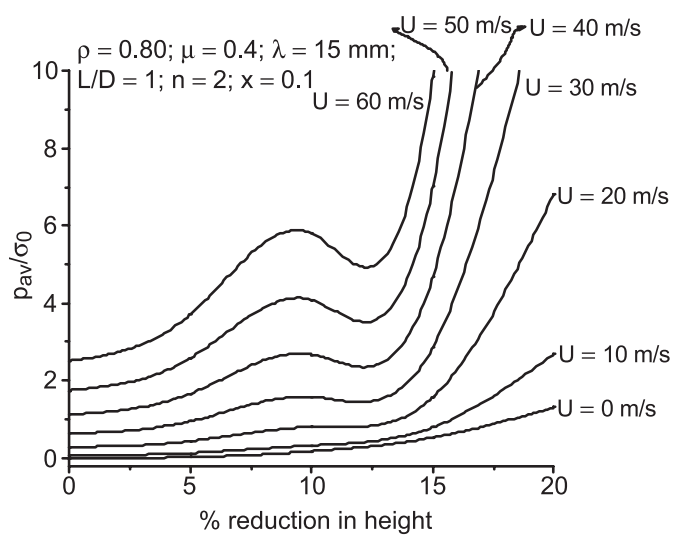

Figure 6. Effect of ram velocity on relative average forging pressure.

\section{Conclusion}

During forging of powder preforms, the mode of deformation is quite different from wrought materials and it is a function of both density and hydrostatic stress. During high-speed forging of sintered materials the main controlling factors are the deformation speed, the amount of interfacial friction between the die-work piece interface, the initial density of the preform and $L / D$ (slenderness) ratio of the preform. The relative average forging pressure of the sinterforged preform increases with increase in initial relative density of the preform, coefficient of friction and ram velocity. Different dimensions of the preform (i.e. $L / D$ ) have been taken here for the analysis of deformation behaviour. The problem of relative theoretical die load and effect of slenderness ratio (i.e. $L / D$ ) have been investigated by applying an appropriate interfacial friction law and plasticity theory of porous metals.

It is also concluded that the effect of die velocity on the relative average forging pressure is not negligible during high-speed sinter forging and must be considered while estimating the deformation load. The inertial forces encountered are functions of processing parameters and deformation characteristics of the sintered material. Evidently, such dynamic effects within the forged material itself are important for very fast forging operations.

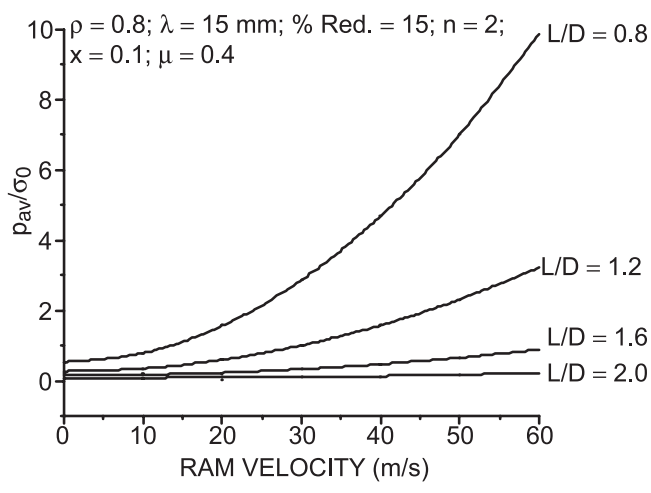

Figure 7. Effect of ram velocity on average relative forging pressure. 


\section{List of symbols}

$\begin{array}{ll}a & \text { amplitude; } \\ D & \text { diameter of the preform; } \\ J_{2}^{\prime} & \text { second invariant of deviatoric stress; } \\ k & \text { constant equal to 2 in yield criterion; } \\ L & N \lambda=\text { length of the preform; } \\ n & \text { constant quantity much greater than } 1 ; \\ p_{a v} & \text { ram pressure; } \\ R & \text { radius of the preform; } \\ R_{m} & \text { radius of the sticking zone; } \\ R, \theta, Y & \text { cylindrical co-ordinates; } \\ U & \text { ram velocity; } \\ \varepsilon_{R}, \varepsilon_{\theta}, \varepsilon_{y} & \text { principal strain increment; } \\ \eta & \text { constant and a function of } \rho \text { only; } \\ \lambda & \text { wavelength; } \\ \mu & \text { coefficient of friction; } \\ \rho & \text { relative density of the preform; } \\ \rho_{r} & \text { real density of the preform; } \\ \sigma_{0} & \text { yield stress of the non-work hardening matrix metal; } \\ \tau & \text { shear stress. }\end{array}$

\section{References}

Agrawal M, Kumar S, Jha A K 1999 High speed forging of hollow metal preforms. J. Inst. Eng. (India) 80: $8-15$

Deryagin B V 1952 What is friction? Izd. Akad. Nauk, USSR

Jha A K, Kumar S 1983 Forging of metal-powder preforms. Int. J. Mach. Tool Design Res. 23: 201-206

Jha A K, Kumar S 1987 Interfacial friction during cold processing of metal-powder-preforms. Proceedings of the International Tribology Conference (The Institution of Engineers) p 346

Jha A K, Kumar S 1996 Dynamic effect during high speed sinter-forging process. Int. J. Mach. Tool Des. Res. 36: 1109-1113

Jha A K, Kumar S 1997 Investigation into high-speed forging of sintered copper powder strips. $J$. Mater. Proc. Technol. 71: 394

Kumar S, Jha A K, Singh R K, Singh S 2001 Sinter preforms add better value to aerospace components. J. Inst. Eng. (India) 82: 1-6

Ramakrishnan P 1980 Proc. Int. Seminar on Metal Working Technology Today and tomorrow, National Inst. of Foundary and forge technology Ranchi, 43

Rooks B W 1974 The effect of die temperature on metal flow and die-wear during high-speed hot forging. Proc. 15th Int. MTDR conference, Birmingham, pp 487-493

Sutradhar G, Jha A K, Kumar S 1995 Cold forging of sintered iron-powder preforms. J. Mater. Proc. Technol. 52: 369-386 